\title{
Simulation Analysis of DDS Signal in Dynamic Environment
}

\author{
Yang Liu, Gang Fu \& Dongxu Zhu \\ China Satellite Maritime Tracking and Control Department, Jiangyin, 214431, China
}

\begin{abstract}
Spread spectrum technology is an information transmission technology, which channel bandwidth occupied by the information that far exceed the minimum bandwidth necessary for transmission; the spectrum is expanded by a separate code sequence is complete, the coding and modulation method to achieve regardless of the data transfer of information; at the receiving end with the same code sequence synchronized to receive relevant information despreading and recover the data transmission. This paper focuses on the basic characteristics of a spread spectrum signal, and on the dynamic simulation environment monitoring and control of the spread spectrum signal acquisition and tracking, establish overall concept and theoretical foundation for the spread spectrum telemetry signal processing.
\end{abstract}

KEYWORD: DSSS signal; simulation; dynamic

\section{INTRODUCTION}

With the new monitoring and control technologies emerging, intelligent measurement and control system to the wide-band, high-speed, real-time, comprehensive and other aspects of development, the formation of an integrated monitoring and control network over the horizon (SPILLARD C.A, 2012). Dynamic seriously low SNR spread spectrum telemetry technology to improve the comprehensive ability aerospace monitoring and control systems, measurement and control technology is a priority of development (R.A. Iltis, 2010). DSSS transmission control technology is an information technology, this paper focuses on the basic characteristics of a spread spectrum signal, and on the dynamic simulation environment monitoring and control of the spread spectrum signal acquisition and tracking, establish overall concept and theoretical foundation for the spread spectrum telemetry signal processing (R.A. Iltis, 2013).

\section{CHARACTERISTICS OF DSSS SIGNAL}

S-band spread spectrum signals using BPSK modulation measurement and control system, is not considered a variety of error can be written as:

$$
\operatorname{Re}_{R F}=\sqrt{2 P} P N(t) D(t) \cos \left(2 \pi f_{R F} t\right)
$$

Spreading PN code sequence length is 1023, the code rate is $1.023 \mathrm{MHz} * \mathrm{n},(\mathrm{n}=1,2 \ldots 10)$. Pseudo code through two 10-bit shift register G1 and G2 produce, G1 and G2 produce a length of the maximum length of the pseudo-code 1023. Pseudo-code is a direct output from the delay in the G1 and G2 of the output of the XOR result, it produces shown in Figure 1:

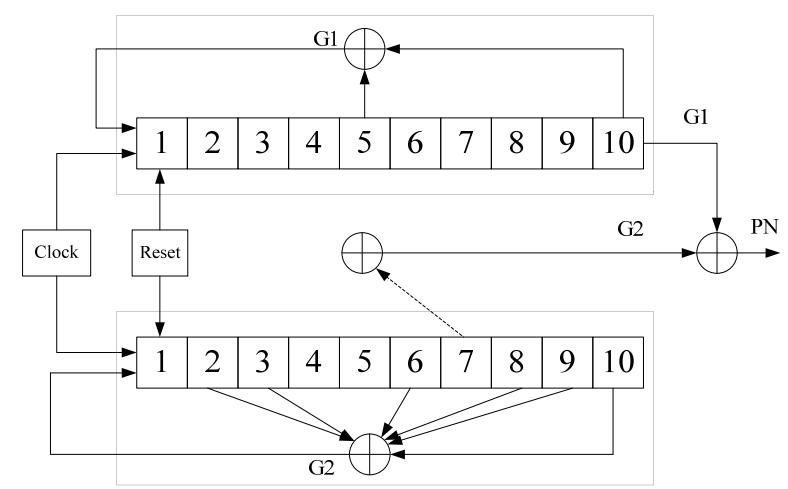

Figure 1. Pseudo-code generator

Pseudo-code correlation function:

$$
R(\tau)=\int_{0}^{T} C(t) C(t+\tau) d t
$$

Pseudo-code autocorrelation in Figure 2 (a) below. Receiver so that local PN code and the received signal PN code phase alignment, to obtain higher processing gain, and can use this feature properly 
aligned pseudo code ranging. The use of pseudocode cross-correlation can be realized multiple access communication, cross-correlation shown in Figure 2 (b) below:

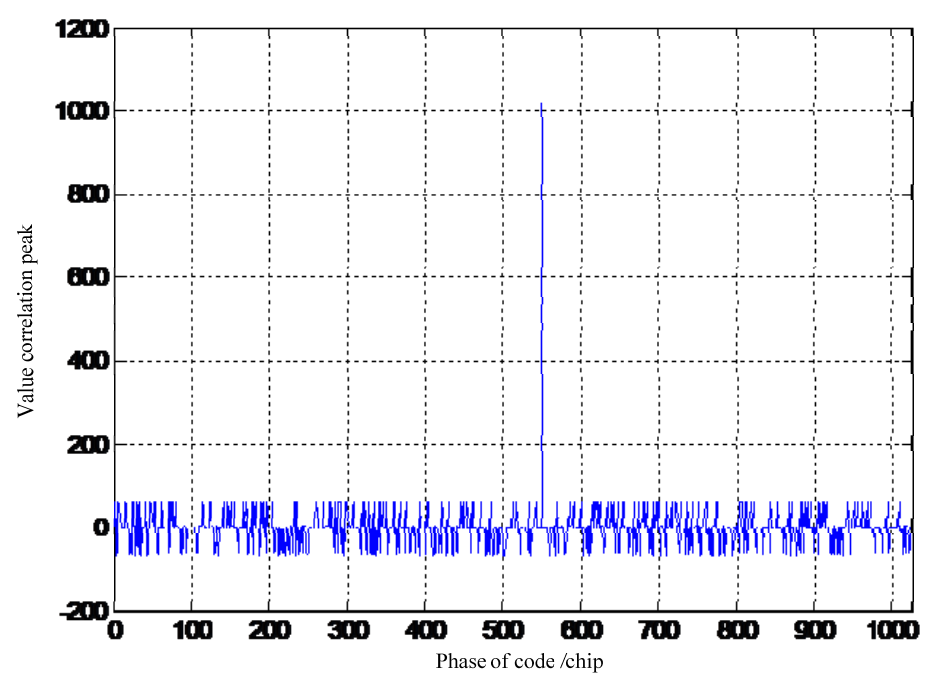

(a). PN autocorrelation

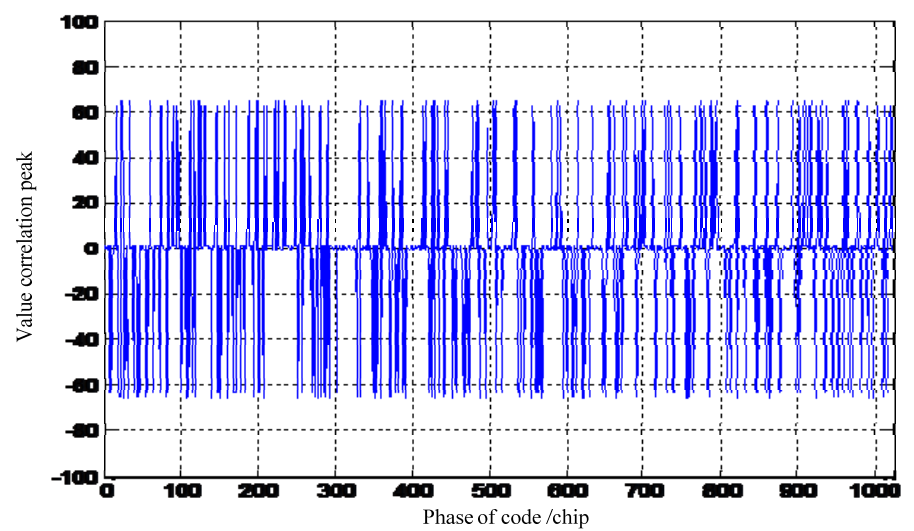

(b). Pseudo-code cross-correlation

Figure 2. Pseudo-code correlation

The front-end filter bandwidth autocorrelation value signal is expressed as follows:

$R_{\mathrm{BL}}(\tau)=\frac{1}{\pi}(\tau+1) \operatorname{Si}[2 \pi \mathrm{a}(\tau+1)]+\frac{1}{2 \pi^{2} \mathrm{a}} \cos [2 \pi \mathrm{a}(\tau+1)]+\frac{1}{\pi}(\tau-1) \operatorname{Si}[2 \pi \mathrm{a}(\tau-1)]$ $=\frac{1}{2 \pi^{2} \mathrm{a}} \cos [2 \pi \mathrm{a}(\tau-1)]-\frac{2}{\pi} \operatorname{Si}[2 \pi \mathrm{a} \tau]-\frac{1}{\pi^{2} \mathrm{a}} \cos (2 \pi \mathrm{a} \tau)$

Front-end filter bandwidth normalized autocorrelation value relationship as shown in Figure 3:

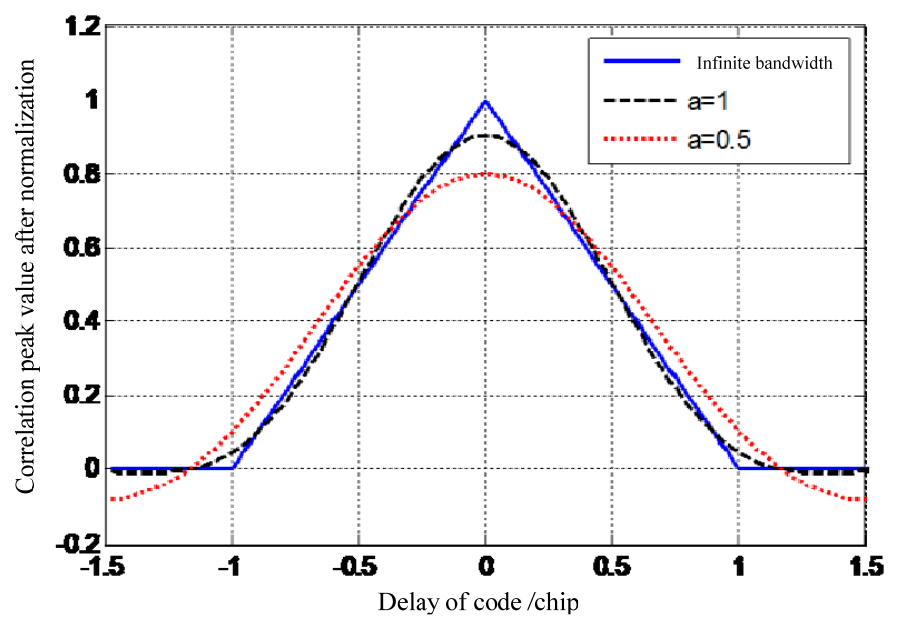

Figure 3. The front-end filter bandwidth normalized autocorrelation value relationship

\section{DYNAMIC EFFECTS OF SPREAD SPECTRUM SIGNAL ACQUISITION}

In remote sensing and control process, when there is relative movement between the control station and monitoring and control target, the received signal carrier frequency offset occurs. Whether motion transmitter or receiver motion, as long as the transmitter and receiver relative radial velocity is, the reception frequency becomes:

$f_{r}=\left(1 \pm \frac{v}{c}\right) f_{R F}$

Defined receive frequency and transmit frequency difference between the frequency of the Doppler frequency, namely:

$$
f_{d}=f_{r}-f_{R F}= \pm \frac{v}{C} f_{R F}
$$

Wherein, when the transmitter and the receiver close to each other to take a positive sign, away from each other when the negative sign, the following symbol selection with this. As can be seen from equation (5), the higher the signal frequency, the larger the Doppler shift. Doppler shift amount by the change of mode is obtained containing the Doppler shift of the received signal is:

$\operatorname{Re}(t)=\sqrt{2 P} P N(t) D(t) \cos \left[2 \pi\left(f_{R F}+f_{d}\right) t+\varphi\right]$

Downconverted signal is filtered in-phase, quadrature amount:

$$
\begin{aligned}
& I(k)=\sqrt{2 P} P N(k) D(k) \cos \left(2 \pi f_{d} T_{s} k+\varphi\right) \\
& Q(k)=-\sqrt{2 P} P N(k) D(k) \sin \left(2 \pi f_{d} T_{s} k+\varphi\right)
\end{aligned}
$$

The N-point correlation accumulation results:

$I^{\prime}(n)=\sum^{N} I(k)=\sqrt{2 P} R_{P N}(\tau)\left|\frac{\sin \pi f_{d} N T_{s}}{\pi f_{d} N T_{s}}\right| D(n) \cos \left(2 \pi f_{d} N T_{s} n+\varphi\right)(9)$ 
$Q^{\prime}(n)=\sum^{N} Q(k)=\sqrt{2 P} R_{P N}(\tau)\left|\frac{\sin \pi f_{d} N T_{s}}{\pi f_{d} N T_{s}}\right| D(n) \sin \left(2 \pi f_{d} N T_{s} n+\varphi\right)$

Correlation peak value is:

$S=\sqrt{\left[\left(I^{\prime}(n)\right)^{2}+\left(Q^{\prime}(n)\right)^{2}\right]}=\sqrt{2 P} R_{P N}(\tau) \cdot\left|\frac{\sin \left(\pi f_{d} N T_{s}\right)}{\pi f_{d} T_{s}}\right|$

From equation (11), the Doppler frequency related cumulative impact on the result is to make the correlation peak decreased $\left|\frac{\sin \left(\pi f_{d} N T_{s}\right)}{\pi f_{d} N T_{s}}\right|$.

\section{EFFECT OF NOISE ON THE SPREAD SPECTRUM SIGNAL}

By the quadrature modulation method can be narrowband additive white gaussian noise:

$N(t)=I_{N}(t) \cos \left[2 \pi\left(f_{c}+f_{d}\right) t\right]+Q_{N}(t) \sin \left[2 \pi\left(f_{c}+f_{d}\right) t\right]$

$\mathrm{n}(\mathrm{t})$ is divided into two branches, obtained by down-conversion and low-pass filtered:

$N_{I}(t)=I_{N}(t) \cos \left(2 \pi f_{d} t\right)+Q_{N}(t) \sin \left(2 \pi f_{d} t\right)$

$N_{Q}(t)=-I_{N}(t) \sin \left(2 \pi f_{d} t\right)+Q_{N}(t) \cos \left(2 \pi f_{d} t\right)$

The output of the above two formulas sampled as follows:

$N_{I}(k)=I_{N}\left(k T_{s}\right) \cos \left(2 \pi f_{d} k T_{s}\right)+Q_{N}\left(k T_{s}\right) \sin \left(2 \pi f_{d} k T_{s}\right)$

$N_{Q}(k)=-I_{N}\left(k T_{s}\right) \sin \left(2 \pi f_{d} k T_{s}\right)+Q_{N}\left(k T_{s}\right) \cos \left(2 \pi f_{d} k T_{s}\right)$

The output of two sampling results associated with the local code after the operation were:

$N_{I}^{\prime}(k)=\frac{1}{N} \sum_{k=1}^{N} P N(k-\tau)\left[I_{N}\left(k T_{s}\right) \cos \left(2 \pi f_{d} k T_{s}\right)+Q_{N}\left(k T_{s}\right) \sin \left(2 \pi f_{d} k T_{s}\right)\right]$

$N_{Q}^{\prime}(k)=\frac{1}{N} \sum_{k=1}^{N} P N(k-\tau)\left[-I_{N}\left(k T_{s}\right) \sin \left(2 \pi f_{d} k T_{s}\right)+Q_{N}\left(k T_{s}\right) \cos \left(2 \pi f_{d} k T_{s}\right)\right]$

Because the pseudo-noise code and independent pseudo-code value of \pm 1 , so the accumulator output noise mean and variance are:

$$
\begin{aligned}
E\left(N_{I}^{\prime}\right) & =E\left\{\frac{1}{N} \sum_{k=1}^{N} P N(k-\tau)\left[I_{N}\left(k T_{s}\right) \cos \left(2 \pi f_{d} k T_{s}\right)+Q_{N}\left(k T_{s}\right) \sin \left(2 \pi f_{d} k T_{s}\right)\right]\right\} \\
& =\frac{1}{N} \sum_{1}^{N}\left\{E[P N(k-\tau)] \cdot E\left[I_{N}\left(k T_{s}\right)\right] \cdot E\left[\cos \left(2 \pi f_{d} k T_{s}\right)\right]\right\} \\
& +\frac{1}{N} \sum_{1}^{N}\left\{E[P N(k-\tau)] \cdot E\left[Q_{N}\left(k T_{s}\right)\right] \cdot E\left[\sin \left(2 \pi f_{d} k T_{s}\right)\right]\right\} \\
& =0
\end{aligned}
$$

$$
\begin{aligned}
D\left(N_{I}^{\prime}\right) & =D\left\{\frac{1}{N} \sum_{k=1}^{N} P N(k-\tau)\left[I_{N}\left(k T_{s}\right) \cos \left(2 \pi f_{d} k T_{s}\right)+Q_{N}\left(k T_{s}\right) \sin \left(2 \pi f_{d} k T_{s}\right)\right]\right\} \\
& =\frac{1}{N} \sum_{1}^{N}\left\{D[P N(k-\tau)] \cdot D\left[I_{N}\left(k T_{s}\right)\right] \cdot D\left[\cos \left(2 \pi f_{d} k T_{s}\right)\right]\right\} \\
& +\frac{1}{N} \sum_{1}^{N}\left\{D[P N(k-\tau)] \cdot D\left[Q_{N}\left(k T_{s}\right)\right] \cdot D\left[\sin \left(2 \pi f_{d} k T_{s}\right)\right]\right\} \\
& =\frac{\sigma^{2}}{N}
\end{aligned}
$$

In summary, the accumulator output noise are zero mean and variance for a random Gaussian noise 3 , the statistical properties of the carrier frequency Doppler irrelevant. Simulation of the signal shown in Figure 4.

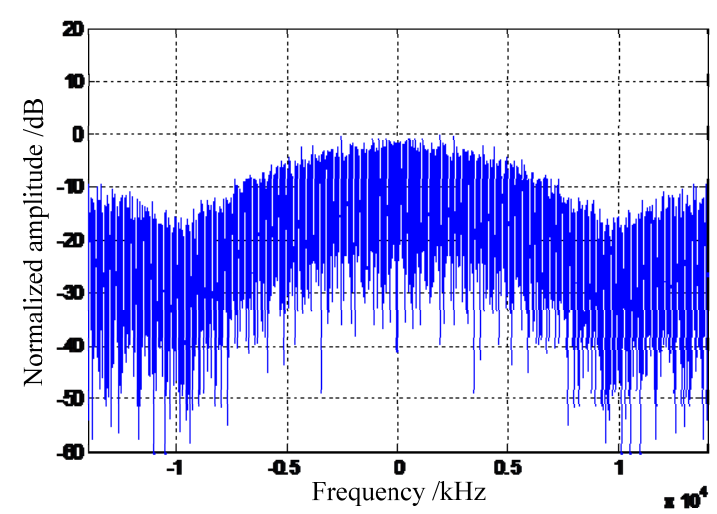

(a). original signal frequency spectrum

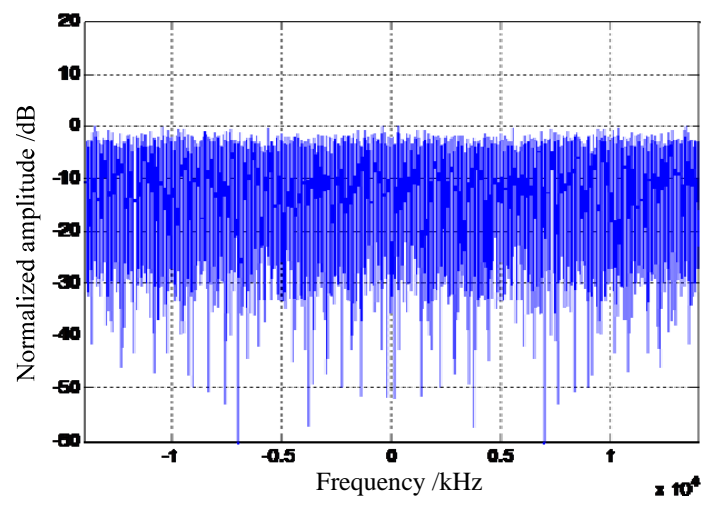

(b). signal frequency spectrum after the addition of the noise

Figure 4. The impact of the spread spectrum signal plus noise

As can be seen from the figure, the actual reception, a spread spectrum telemetry signal in the noise being completely covered.

\section{SUMMARY}

In this paper, the basic composition and properties of the pseudo-code spreading and Control System are described, analyzed the effects of various factors on the monitoring and control of dynamic measurement and control the spread spectrum signal acquisition and tracking, and establish overall concept and theoretical foundation for the spread spectrum telemetry signal processing. 


\section{REFERENCES}

R.A.Iltis.An EKF-based joint estimator for interference, multipath, and code delay in a DS spread-spectrum receiver[J],IEEE Transactions on communications.2013:12881299.

R.A.Iltis.Joint estimation of PN code delay and multipath using the Extended Kalman Filter[J],IEEE Transactions on communications.2010:1677-1685.

SPILLARD C.A serial-parallel FFT corrugators for PN code acquisition from LEO satalites[J]. IEEE Transactions on communications, 2012:446-448. 